\title{
Fabrication of porous alumina ceramics having cell windows with controlled size by PMMA template method
}

\author{
Kazutaka Kamitani ${ }^{1}$, Takeo Hyodo ${ }^{1}$, Yasuhiro Shimizu ${ }^{2}$, Makoto Egashira ${ }^{2}$ \\ ${ }^{1}$ Graduate School of Science and Technology \\ ${ }^{2}$ Faculty of Engineering \\ Nagasaki University, 1-14 Bunkyo-machi, Nagasaki 852-8521, Japan
}

\section{Corresponding Author}

Yasuhiro Shimizu, Prof. Dr.

Department of Materials Science and Engineering,

Faculty of Engineering,

Nagasaki University

1-14 Bunkyou-machi, Nagasaki 852-8521, Japan

TEL: +81-95-819-2642

FAX: $+81-95-819-2643$

E-mail: shimizu@nagasaki-u.ac.jp 


\begin{abstract}
Highly porous alumina compacts have been prepared by filling alumina slurry into opening of a specially designed template block prepared by cross-linked ( $\mathrm{Cl}-$ ) polymethylmethacrylate (PMMA) microspheres (mean particle size: $98.6 \mu \mathrm{m}$ ) under reduced pressure, followed by firing to remove the template and then sintering at $1600^{\circ} \mathrm{C}$. The template blocks were prepared by utilizing cl-PMMA and acetone solution dissolving noncrosslinked ( $\mathrm{NCl}$-) PMMA as an adhesive to make partial connection among the cl-PMMA microspheres. Furthermore, the effects of the addition of other chemicals (two types of triblockcopolymers and a nonionic surfactant) to the Ncl-PMMA adhesive solution were tested to improve the wettability of the solution to $c l$-PMMA and then to control the cell windows among the cl-PMMA-originated cells after the firing. By employing the template block prepared with the adhesive solution containing an appropriate amount of triblockcopolymer, a highly porous alumina compact having an open porosity of $77.2 \%$, a mean cell window diameter of $28.8 \mu \mathrm{m}$ and strong alumina skeleton could be fabricated.
\end{abstract}

\title{
Keywords
}

Porous alumina, Polymethylmethacrylate, Porosity, Template method 


\section{Introduction}

Recently, there has been an increasing interest in the applications of porous ceramics as filters, insulators, catalyst supports, bone replacements and membrane reactors and so on [1-7]. High porosity and optimization of pore size distribution of the porous ceramics are very essential for the enhancement of the diffusivity of various gaseous and liquid substances through their porous structure. These microstructures are largely dependent on their preparation methods. However, it is difficult to realize high open porosities by conventional methods because of insufficient control of interconnection among cells. Therefore, many researchers have been studied and reported various preparation methods, such as pore-forming procedures [8-10], a polymeric sponge replication [11-14], a gel-casting [15-17], preceramic polymers [18, 19], template [20-23] and sol-gel methods [24], in order to achieve high open porosity and well-ordered porous structure. Among them, Kim et al. have succeeded in preparing well-ordered porous ceramics (mean pore size: ca. $320 \mathrm{~nm}$ ) by a physical template method employing polystyrene latex spheres (mean particle size: $400 \mathrm{~nm}$ ) [20].

Recently, we have also prepared various porous films by utilizing a modified sol-gel technique employing polymethylmethacrylate (PMMA) microspheres as a template [22-26]. These porous films have well-ordered spherical pores with sub-micron size, which improved gas reactivity and diffusivity. Therefore, they showed excellent sensing properties to $\mathrm{H}_{2}, \mathrm{NO}_{\mathrm{x}}, \mathrm{H}_{2} \mathrm{~S}$ and inflammable gases [22-26]. However, it is very difficult to form much larger spherical pores with a diameter of several ten microns by this method, because the precursors of ceramic materials cannot be densely filled into large voids among the large microsphere templates. Even if the ceramic skeletons are obtained after the decomposition of the PMMA microspheres by firing, the mechanical strength is terribly low. In addition, the size control of spaces connecting among these spherical pores is also extremely difficult. This is because the connections among PMMA microspheres are easily cut off by the addition of the slurry or the sol solution and thus thin films with different thicknesses are formed among PMMA microspheres.

In this study, therefore, attempts were made to establish the preparation conditions of porous alumina compacts having well-developed and well-controlled spherical PMMA-originated cells and cell windows among them as well as high mechanical strength, by utilizing a specially designed PMMA template method. Crosslinked ( $c l$-) PMMA microspheres were used as a template for spherical PMMA-originated cells and these $\mathrm{cl}$-PMMA microspheres were connected each other at neck parts in advance by the acetone solution dissolving noncrosslinked ( $\mathrm{NCl}$-) PMMA used as an adhesive to form cell windows among the spherical PMMA-originated cells. Effects of the additive amounts of Ncl-PMMA and other chemicals (two types of triblockcopolymers and a nonionic surfactant) to the Ncl-PMMA adhesive solution on the open porosity and the size of cell windows of porous alumina compacts have been investigated. 


\section{Experimental procedure}

\subsection{Preparation of PMMA template blocks}

$0.3 \mathrm{~g}$ of cross-linked (cl-) PMMA microspheres with a mean particle size of $96.7 \mu \mathrm{m}$ (MR-90G, Soken Chem. \& Eng. Co., Ltd.) as a spherical-pore former was packed in a glass pipe (inside diameter: $12 \mathrm{~mm}$, height: $15 \mathrm{~mm}$ ) set vertically on a glass board. Acetone-based adhesive solutions used for the connection of neck parts of the packed cl-PMMA microspheres were prepared by the dissolution of given amounts of non-cross-linked ( $\mathrm{NCl}$-) PMMA microspheres with a mean particle size of $400 \mathrm{~nm}$ (MP-1451, Soken Chem. \& Eng. Co., Ltd.) in $20 \mathrm{ml}$ acetone (Kishida Chem. Co., Ltd.). $250 \mu \mathrm{l}$ of the Ncl-PMMA adhesive solution was permeated into the openings of the packed cl-PMMA microspheres. In some cases, one of the following three chemicals, triblockcopolymers formed from polyethylene oxide (EO) and polypropylene oxide (PO), P-123 $\left(\mathrm{EO}_{20} \mathrm{PO}_{70} \mathrm{EO}_{20}, \mathrm{BASF}\right.$ Corp.) and PE6400 ( $\mathrm{EO}_{13} \mathrm{PO}_{30} \mathrm{EO}_{13}$, BASF Corp.) as an additive and a general surfactant, Triton $\mathrm{X}-100\left(\mathrm{C}_{14} \mathrm{H}_{22} \mathrm{O}\left(\mathrm{C}_{2} \mathrm{H}_{4} \mathrm{O}\right)_{\mathrm{n}}, \mathrm{n}=9\right.$-10, Sigma-Aldrich Co., Ltd.), was added to the Ncl-PMMA adhesive solution. After drying at room temperature for $12 \mathrm{~h}$, a cl-PMMA template block, in which neck parts among cl-PMMA microspheres were connected with a thin Ncl-PMMA film, was obtained in the glass pipe. The $c l$-PMMA template block (PTB) is expressed as PTBmSn, where $\mathrm{S}$ represents the kind of additive (P: P-123, PE: PE6400, and T: Triton X-100) to the Ncl-PMMA adhesive solution and $m$ and $n$ stand for the amounts of the Ncl-PMMA (g) added to the $20 \mathrm{ml}$ acetone and the chemical added in the Ncl-PMMA adhesive solution (g), respectively. PTBm represents the block fabricated by using the Ncl-PMMA adhesive solution without any chemicals.

\subsection{Preparation of porous alumina compacts}

An alumina slurry was prepared by the following procedure: $15 \mathrm{~g}$ of a high purity alumina powder with an average particle size of $0.1 \mu \mathrm{m}$ (TM-DAR: Taimei Chem. Co., Ltd.) and $0.5 \mathrm{~g}$ of P-123 were mixed with $20 \mathrm{ml}$ of deionized water. Then the resultant mixture was subjected to ball milling at a rotation speed of $400 \mathrm{rpm}$ for $30 \mathrm{~min}$ to make an uniform slurry. $\mathrm{PTBmSn}$ prepared was removed from the glass pipe and then was set on a filter (quantitative filter paper, space size: 1.0 $\mu \mathrm{m}$ ). Thereafter, $1.0 \mathrm{ml}$ of the alumina slurry was added from the foreside of PTBmSn, and then the mixture was left stand for $6 \mathrm{~h}$ under vacuum. After the alumina slurry was impregnated into the openings of PTBmSn, it was dried at $45^{\circ} \mathrm{C}$ for $12 \mathrm{~h}$, and then a green alumina-PMMA compact was obtained. If it is assumed that PMMA microspheres used would have the same diameter, the maximum filling fraction of PMMA microspheres can be calculated theoretically to be $74 \%$. Thus, a theoretical open porosity, which will be formed after the removal of PMMA microspheres by thermal decomposition, is $74 \%$ provided that no shrinkage is expected during subsequent firing and sintering. PMMA and other organic additives in the green compact were thermally decomposed by heating at a heating rate of $0.5^{\circ} \mathrm{C} \mathrm{min}^{-1}$ up to $450^{\circ} \mathrm{C}$ and folding at the same temperature for $0.5 \mathrm{~h}$ in 
air, and it was subsequently heated at a heating rate of $10^{\circ} \mathrm{C} \mathrm{min}^{-1}$ up to $1600^{\circ} \mathrm{C}$ and then sintered for $2 \mathrm{~h}$. Spherical PMMA-originated cells and cell windows among the spherical PMMA-originated cells are expected to be formed after the thermal decomposition at $450^{\circ} \mathrm{C}$. The porous $(\mathrm{pr}-)$ alumina compact obtained is expressed as $p r-\mathrm{A}(\mathrm{PTB} m \mathrm{~S} n)$ or $p r-\mathrm{A}(\mathrm{PTB} m)$, where $\mathrm{PTB} m \mathrm{~S} n$ or $\mathrm{PTB} m$ means a template block used, respectively.

\subsection{Characterization}

Microstructure of the PMMA template blocks and the pr-alumina compacts fabricated was observed by a scanning electron microscope (SEM, JEOL Ltd., JCM-5700). The average diameter of PMMA microspheres and PMMA-originated cells was determined by measuring diameters of about a number of 500 of PMMA microspheres and PMMA-originated cells each in their SEM photographs. The mean diameter of cell windows was determined by measuring diameters of a number of 600 cell windows in their SEM photographs.

Pore size distribution of the pr-alumina compacts was measured by a mercury porosimeter (Micromeritics Instrument Corp., AutoPore IV 9500). The open porosity was calculated by using the pore volume measured by the mercury porosimeter and the true density measured by a multivolume pycnometer (Micromeritics Instrument Corp., 1305). The open porosity, $V p$, of $p r$-alumina compacts was evaluated using Eq. (1):

$$
V p=\frac{V}{V+\frac{1}{\rho}}
$$

where $V$ and $\rho$ is pore volume and the true density measured by the mercury porosimeter and the multivolume pycnometer, respectively.

\section{Result and discussion}

3.1 Effects of the amount of Ncl-PMMA in adhesive solution on microstructure of pr-PTBm compacts

Figure 1 shows SEM photographs of fracture surface of six kinds of as-prepared PTBm. As for PTB0, cl-PMMA microspheres with different particle sizes in the range of $58 \sim 110 \mu \mathrm{m}$ were closely packed. But adhesion force among cl-PMMA microspheres is supposed to be very weak and these contact points are considered to be cut off very easily during the subsequent impregnation of the alumina slurry. On the other hand, several numbers of circular thin films less than $20 \mu \mathrm{m}$ in diameter were observed on the surface of cl-PMMA microspheres in the case of PTB0.1, indicating 
the existence of Ncl-PMMA thin films at the neck parts among $c l$-PMMA microspheres as adhesives. Thus, $c l$-PMMA microspheres in PTB0.1 were connected concretely by the Ncl-PMMA thin films. It is obvious that both the number and the size of the Ncl-PMMA thin films at the surface of cl-PMMA microspheres increased with an increase in the amount of Ncl-PMMA in the Ncl-PMMA adhesive solution, $m$. But too much Ncl-PMMA was added, the Ncl-PMMA thin films protruded from the neck parts among cl-PMMA microspheres, as shown in Figs. 1(e) and 1(f), implying the formation of irregular-shaped and -sized cell windows among cl-PMMA microspheres-originating cells in resulting $p r-\mathrm{PTBm}$ compacts after the removal of Ncl-PMMA thin films and cl-PMMA microspheres by firing.

Figure 2 shows SEM photographs of fracture surface of six kinds of pr-A(PTBm) compacts. From these SEM photographs, the formation of a large number of spherical cells could be confirmed for all the $p r-\mathrm{A}(\mathrm{PTBm})$ compacts. The size of these cells was not uniform and varied in a certain range $(46 \sim 96 \mu \mathrm{m})$, but they reflected well the spherical shape of original cl-PMMA microspheres used as a template. The average size of spherical cells for pr-A(PTB0) was $86.8 \mu \mathrm{m}$. In the addition, the average size of spherical cells for other pr-A(PTBm) compacts were showed almost the similar value, for example, $87.5 \mu \mathrm{m}$ for $p r-\mathrm{A}(\mathrm{PTB} 0.1), 88.5 \mu \mathrm{m}$ for $p r-\mathrm{A}(\mathrm{PTB} 0.5)$ and $87.2 \mu \mathrm{m}$ for pr-A(PTB1.5). The shrinkage ratio of pr-alumina compacts was also almost similar value though the amounts of Ncl-PMMA added were different. As for pr-A(PTBO) fabricated without Ncl-PMMA, cell windows were scarcely observed on the inner wall of these spherical cells, and the spherical cells existed obviously as closed cells. But, when the РTB0.1, in which neck parts among cl-PMMA microspheres were connected by the Ncl-PMMA thin films, was used as a template, the formation of some cell windows with a diameter of ca. $15.5 \mu \mathrm{m}$ was observed on the inner wall of spherical cells, as sown in Fig. 2(b). From the comparison of SEM photographs shown in Fig. 1(b) and Fig. 2(b), it is confirmed that the pre-connection of cl-PMMA microspheres by the Ncl-PMMA thin films at their neck parts employed in the present study is very effective for the formation of cell windows among spherical cells and then achieving high open pore volume in pr-A(PTB0.1) compacts. The size of cell windows tended to increase with increasing the $m$ values in PTBm used as a template, e.g., ca. $15.5 \mu \mathrm{m}$ for $p r-\mathrm{A}(\mathrm{PTB} 0.1)$ and ca. $18.3 \mu \mathrm{m}$ for $p r-\mathrm{A}(\mathrm{PTB} 0.5)$, and then the number of cell windows increased as well. However, the morphology of pr-A(PTBm) changed significantly when the $m$ value of the PTBm templates exceeded 1.0. As for pr-A(PTB1.0) and pr-A(PTB1.5), namely, the formation of large ellipsoidal and/or irregular-shaped cell windows was confirmed and alumina skeletons (i.e. the inner walls of spherical pores) were destroyed in some areas, probably due to weak mechanical strength of alumina walls around large cell windows.

Figure 3 shows pore size distributions of $p r-\mathrm{A}(\mathrm{PTBm})$ compacts. Considering the measurement principle of the mercury porosimeter, the pore size distributions measured can be regarded as the diameter of the narrowest and/or bottleneck pores, i.e. the diameter of cell windows 
on the inner wall of $c l$-PMMA-originated cells shown in Fig. 2. Actually, the pore diameter at the peak of pore volume is well coincident with the average diameter of cell windows for each pr-A(PTBm) compact, except for pr-A(PTB0), which was measured by using their SEM photographs (see Fig. 4). The peak diameter at the maximum pore volume for pr-A(PTB0) was $18.5 \mu \mathrm{m}$, and the pore volume was the smallest among all the pr-A(PTBm) compacts. This is because, in the case of $p r-\mathrm{A}(\mathrm{PTB})$ ), the neck parts among cl-PMMA microspheres in the template block were not connected by Ncl-PMMA thin films in advance, and therefore it is considered that only a limited number of cell windows can be formed by the shrinkage of the thin alumina walls existing among cl-PMMA-originated cells during the sintering. On the other hand, the pore diameter at the maximum pore volume of $p r-\mathrm{A}(\mathrm{PTBm})(m>0)$ increased with an increase in $m, 15.4$ $\mu \mathrm{m}$ for $p r-\mathrm{A}(\mathrm{PTB} 0.1), 18.5 \mu \mathrm{m}$ for $p r-\mathrm{A}(\mathrm{PTB} 0.3)$ and $19.1 \mu \mathrm{m}$ for $p r-\mathrm{A}(\mathrm{PTB} 0.5)$. This means that the conditions of the Ncl-PMMA thin films existed at the neck parts among cl-PMMA microspheres in the PTBm was extremely important to form the cell windows among spherical large cells and also to control the size of cell windows. Above $\mathrm{m} \geq 1.0$, however, the pore diameter increased drastically and the peak width broadened with increasing $m$, i.e. a larger variation in size of cell windows. This may imply that excess amounts of Ncl-PMMA resulted in the formation of agglomerates at the neck parts among $c l$-PMMA microspheres in PTBm $(m \geq 1.0)$ and then destroyed the alumina skeleton due to a decrease in the mechanical strength of alumina walls among spherical large cells, besides the formation of the large cell windows.

Figure 4 shows variations in both the size of cell windows measured by using SEM photographs and mercury porosimeter and the open porosity of the $p r-\mathrm{A}(\mathrm{PTBm})$ compacts with the amounts of Ncl-PMMA in the Ncl-PMMA adhesive solution. It is obvious that the size of cell windows estimated by the measurement of mercury porosimeter is very close to that measured by using SEM photographs. And the both sizes of cell windows measured by two methods show similar variations against the change in $m$ value. For both cases, the increase in the size of cell windows was very small at $m \leq 0.5$, but beyond that it increased drastically with an increase in $m$. On the other hand, the open porosity of $p r-\mathrm{A}(\mathrm{PTBm})$ compacts increased drastically at $m=0.1$. This means that the introduction of the $\mathrm{Ncl}$-PMMA thin films at the neck parts of $\mathrm{cl}$-PMMA microspheres in the PTBm is very effective in connecting the most spherical large cells and then in enhancing the open porosity. But, the open porosity increase slightly with increasing $m$ up to $m=1.0$ and beyond that it almost saturated at the theoretical value of $74 \%$.

An almost $74 \%$ of open porosity was achieved at $m \geq 1.0$, however mechanical strength of alumina compacts was very weak. Therefore, the preparation conditions of NCl-PMMA thin films have been attempted to control by adding a chemical reagent in the Ncl-PMMA adhesive solution in order to inhibit the destruction of alumina skeleton, while maintaining high open porosity. 


\subsection{Effect of additives to Ncl-PMMA adhesive solution on microstructure of pr-A(PTB0.5Sn) compacts}

One of the following three chemicals, P-123, PE6400 and Triton X-100, was added to the Ncl-PMMA adhesive solution to improve its wettability to cl-PMMA microspheres and then to obtain uniform distribution of Ncl-PMMA thin films among cl-PMMA microspheres and high mechanical strength of resulting template compacts. From the technical viewpoint, it was impossible to measure actually the contact angle of the Ncl-PMMA adhesive solutions with and without additional chemicals on a cl-PMMA microsphere. Thus, a Si wafer, of which surface is also hydrophobic like cl-PMMA microspheres, was used as an alternative substrate. Figure 5 shows variations in contact angle of Ncl-PMMA adhesive solutions with amounts of several chemicals added. The contact angle of the Ncl-PMMA adhesive solution decreased with an increase in the amount of the chemical added for all the cases. In addition, it is also confirmed that the addition of PE6400 or Triton X-100 is effective in improving the wettability of the Ncl-PMMA adhesive solution to the hydrophobic Si substrate, in comparison of that of P-123.

Figure 6 shows SEM photographs of fracture surface of PTB0.5PEn template blocks and pr-A(PTB0.5PEn) compacts. The size of adhesive Ncl-PMMA thin films on the surface of cl-PMMA microspheres remained almost unchanged irrespective of additive amounts of PE6400. However, from Fig. 6 (a-2), (b-2) and (c-2), the size of cell windows tended to increase with increasing the $n$ values in PTB0.5PEn, e.g., ca. $19.6 \mu \mathrm{m}$ for $p r-\mathrm{A}(\mathrm{PTB} 0.5 \mathrm{PE} 0.1)$ and ca. $24.8 \mu \mathrm{m}$ for pr-A(PTB0.5PE0.5). Thus, it is revealed that the mixing of PE6400 to the Ncl-PMMA solution was very effective in increasing the wettability of Ncl-PMMA solution on the surface of $c l$-PMMA microspheres and thus in improving the open porosity and the size of cell windows of $p r-\mathrm{A}(\mathrm{PTB} 0.5 \mathrm{PEn})$ compacts. Other $p r-\mathrm{PTBmSn}$ also showed the similar tendency as $p r-\mathrm{PTB} 0.5 \mathrm{PEn}$, e.g. the size of $p r-\mathrm{A}(\mathrm{PTB} 0.5 \mathrm{~T} n)$ compacts increased with an increase in $n, 19.2 \mu \mathrm{m}$ for $p r-\mathrm{A}(\mathrm{PTB} 0.5 \mathrm{~T} 0.1), 21.2 \mu \mathrm{m}$ for $p r-\mathrm{A}(\mathrm{PTB} 0.5 \mathrm{~T} 0.3)$ and $23.7 \mu \mathrm{m}$ for $p r-\mathrm{A}(\mathrm{PTB} 0.5 \mathrm{~T} 0.5)$. These results show that the control of the wettability of the Ncl-PMMA adhesive solution is very effective in increasing the size of the cell windows. However, the average size of the spherical PMMA-originated cells for pr-A(PTB0.5Sn) compacts was similar to those observed for pr-A(PTBm) compacts, e.g. the average size of the spherical PMMA-originated cells was $87.9 \mu \mathrm{m}$ for $p r-\mathrm{A}(\mathrm{PTB} 0.5 \mathrm{PE} 0.1)$ and $88.7 \mu \mathrm{m}$ for $p r$-A(PTB0.5PE0.5), because all $p r$-alumina compacts were prepared by employing the same PMMA microspheres as a template. Irrespective of the addition of chemicals to the Ncl-PMMA adhesive solution, the shrinkage ratio of resultant $p r$-alumina compacts was almost the similar value. The average size of cell windows of $p r-\mathrm{A}(\mathrm{PTB} 0.5 \mathrm{PEn})$ compacts fabricated in the present study was larger than those of porous ceramics fabricated by using pore-foaming agents [27], while both the compacts showed similar cell size distributions. Thus, The advantage of our template method is easy controllability of cell windows size. 
Figure 7 shows pore size distributions of three kinds of $p r-\mathrm{A}(\mathrm{PTB} 0.5 \mathrm{~S} 0.5)$ compacts. The pore size at the maximum pore volume and the value of the pore volume of $p r-\mathrm{A}(\mathrm{PTB} 0.5 \mathrm{PE} 0.5)$ and $p r-\mathrm{A}(\mathrm{PTB} 0.5 \mathrm{~T} 0.5)$ are larger than those of $p r-\mathrm{A}(\mathrm{PTB} 0.5 \mathrm{P} 0.5)$. In addition, those of all pr-A(PTB0.5S0.5) are much larger than those of pr-A(PTB0.5) (see Fig. 3 (d)). These results confirm the usefulness the addition of chemicals to the Ncl-PMMA adhesive solution to improve its wettability to $c$-PMMA microspheres and then to increase pore volume as well as the size of cell windows. Furthermore, a sub-peak with a smaller pore diameter than that of the main peak was observed in the pore size distribution for each pr-A(PTB0.5S0.5) compact. In addition, the height of the sub-peak increased clearly with an increase in the wettability of the Ncl-PMMA adhesive solution. Figure 8 shows SEM photographs of fracture surface of two kinds of pr-A(PTB0.5S0.5) compacts. The formation of cell windows with smaller diameter was confirmed obviously on the cell's inner walls from the SEM photographs of pr-A(PTB0.5S0.5) (Figs. 6 (c-2), 8 (a) and (b)). Considering the size of both cell windows, therefore, the main- and sub-peaks reflect the larger and smaller cell windows, respectively. However, the formation mechanism of smaller peaks observed is obscure and this is another subject of our future work.

Figure 9 shows variations in both the size of cell windows and the open porosity of pr-A(PTB0.5Sn) measured by mercury porosimeter, with the amounts of chemicals added to Ncl-PMMA adhesive solutions. Both the size of cell windows and the open porosity of pr-A(PTB0.5Sn) increased gradually with an increase in the amount of chemicals added, regardless of the type of chemicals. The open porosity of pr-A(PTB0.5) without the addition of chemicals was $68.8 \%$. But, only a small additive amount of chemicals to Ncl-PMMA adhesive solutions increased the open porosity drastically from $68.8 \%$ for $p r-\mathrm{A}(\mathrm{PTB} 0.5)$ to $73.8 \%$ for pr-A(PTB0.5PE0.1), although a larger amount of Ncl-PMMA was necessary to achieve comparable open porosity without additional chemical (see Fig. 4). As described previously, a larger amount of Ncl-PMMA resulted in the destruction of alumina skeleton (see Fig. 2(e) and (f)), however the addition of chemicals to the Ncl-PMMA adhesive solution could realize higher open porosity only with a small amount of Ncl-PMMA without the destruction of alumina skeleton (see Figs. 6 (c-2), 8 (a) and 8 (b)). These results show that the addition of chemicals to the Ncl-PMMA adhesive solution is very effective in improving the morphology and the size of cell windows and thus in increasing the open porosity, while maintaining certain mechanical strength of porous structure.

The size of cell windows and the open porosity obtained with all pr-A(PTB0.5Sn) are plotted as a function of the contact angle of the Ncl-PMMA adhesive solutions on the Si substrate, as shown in Fig. 10. An almost liner relationship existed between the size of cell windows measured by both SEM photographs and mercury porosimeter and the contact angle for all the data obtained with pr-A(PTB0.5Sn), irrespective of the kinds and the amounts of chemicals added. Namely, the smaller the contact angle, in other words the higher the wettability, the larger the size of cell 
windows. A similar relationship was observed between the open porosity and the contact angle. Thus, the wettability of the Ncl-PMMA adhesive solution to $c$-PMMA microspheres was found to the most important factor for achieving open porous structure of resulting alumina ceramics with sufficient mechanical strength of alumina skeleton.

\section{Conclusion}

Highly porous alumina compacts have been prepared by filling alumina slurry into opening of a PTB under reduced pressure, followed by firing to remove a PTB and then sintering at $1600^{\circ} \mathrm{C}$. As for $p r-\mathrm{A}(\mathrm{PTB} 0)$ fabricated without Ncl-PMMA, cell windows were scarcely observed on the inner wall of these spherical cells. Partial pre-connection among the cl-PMMA microspheres by the Ncl-PMMA adhesive solution improved the open porosity drastically, but an excess addition of Ncl-PMMA to the adhesive solution resulted in the formation of irregular-shaped and -sized cell windows as well as the destruction of alumina skeletons in $p r-\mathrm{A}(\mathrm{PTBm})$, although high open porosity could be achieved. On the other hand, the additions of other chemicals (two types of triblockcopolymers and a nonionic surfactant) to the Ncl-PMMA adhesive solution was very effective in increasing the wettability of Ncl-PMMA adhesive solution to the surface of $c l$-PMMA microspheres and thus in improving the open porosity and the size of cell windows of pr-A(PTBmSn) compacts. Among them, pr-A(PTB0.5PE0.5) showed the largest open porosity of $77.2 \%$ along with a mean cell windows diameter of $28.8 \mu \mathrm{m}$. 


\section{REFERENCE}

[1] Matsusaki M, Yoshida H, Akashi M (2007) Biomaterials 28: 2729

[2] Kim Y, Park C B (2003) Compos Sci Technol 63: 2371

[3] Isobe T, Kameshima Y, Nakajima A, Okada K, Hotta Y (2007) J Eur Ceram Soc 27: 53

[4] Nangrejo M, Bernardo E, Colombo P, Farook U, Ahmad Z, Stride E, Edirisinghe M (2009) Mater Lett 63: 483

[5] Mear F, Yot P, Viennois R, Ribes M (2007) Ceram Int 33: 543

[6] Zou C, Weng W, Deng X, Cheng K, Liu X, Du P, Shen G, Han G (2005) Biomaterials 26: 5276

[7] Zampieri A, Colombo P, Mabande G T P, Selvam T, Schwieger W, Scheffler F (2004) Adv Mater 16 (9-10): 819

[8] Tian T, Jiang D, Zhang J, Lin Q (2008) Mater Sci Eng C 28: 51

[9] Jo I, Shin K, Soon Y, Koh Y, Lee J, Kim H (2009) Mater Lett 63: 1702

[10] Zhang Y, Liang H, Zhao C Y, Liu Y (2009) J Mater Sci 44: 931

[11] He X, Zhou X, Su B (2009) Mater Lett 63: 830

[12] Wang X, Ruan J, Chen Q (2009) Mater Res Bull 44: 1275

[13] Maire E, Colombo P, Adrien J, Babout L, Biasetto L (2007) J Eur Ceram Soc 27: 1973

[14] Sifontes A B, Urbina M, Fajardo F, Melo L, Mediavilla M, Carrio'n N, Brito J L (2009) J Mater Sci 44: 4507. Doi: 10. 1007/ s10853-009-3693-3

[15] Zhang F, Kato T, Fuji M, Takahashi M (2006) J Eur Ceram Soc 26: 667

[16] Mao X, Shimai S, Wang S (2008) J Eur Ceram Soc 28: 217

[17] Ortega F S, Sepulveda P, Pandolfelli V C (2002) J Eur Ceram Soc 22: 1395

[18] Zeschky J, Goetz-Neunhoeffer F, Neubauer J, Jason Lo S H, Kummer B, Scheffler M, Greil P (2003) Compos Sci Technol 63: 2361

[19] Colombo P (2008) J Eur Ceram Soc 28: 1389

[20] Kim Y, Kim C, Yi J (2004) Mater Res Bull 29: 2103

[21] Wang H, Zheng S, Li X, Kim D (2005) Microporous Mesoporous Mater 80: 357

[22] Takakura Y, Hyodo T, Shimizu Y, Egashira M (2008) IEE J Trans 128: 137

[23] Hieda K, Hyodo T, Shimizu Y, Egashira M (2008) Sens Actuator B 133: 144

[24] Sasahara K, Hyodo T, Shimizu Y, Egashira M (2004) J Eur Ceram Soc 24: 1961

[25] Seh H, Hyodo T, Tuller H L (2005) Sens Actuator B 108: 547

[26] Nonaka S, Hyodo T, Shimizu Y, Egashira M (2007) Chem Sens 23: 121

[27] Mao X, Wang S, Shimai S (2008) Ceram Int 34: 107 
Figure captions

Fig. 1 SEM photographs of fracture surface of (a) PTB0, (b) PTB0.1, (c) PTB0.3, (d) PTB0.5, (e) PTB1.0 and (f) PTB1.5.

Fig. 2 SEM photographs of fracture surface of (a) pr-A(РTB0), (b) pr-A(РTB0.1), (c) $p r-\mathrm{A}(\mathrm{PTB} 0.3)$, (d) $p r-\mathrm{A}(\mathrm{PTB} 0.5),(\mathrm{e}) p r-\mathrm{A}(\mathrm{PTB} 1.0)$ and (f) $p r-\mathrm{A}(\mathrm{PTB} 1.5)$.

Fig. 3 Pore size distributions of (a) pr-A(PTB0), (b) pr-A(РTB0.1), (c) pr-A(PTB0.3), (d) $p r-\mathrm{A}(\mathrm{PTB} 0.5)$, (e) $p r-\mathrm{A}(\mathrm{PTB} 1.0)$ and (f) $p r-\mathrm{A}(\mathrm{PTB} 1.5)$.

Fig. 4 Variations in size of cell windows measured by using SEM photographs and mercury porosimeter and open porosity in $p r-\mathrm{A}(\mathrm{PTBm})$ with $m$ values.

Fig. 5 Variations in contact angle of Ncl-PMMA adhesive solutions on a Si wafer with amounts of several chemicals added.

Fig. 6 SEM photographs of fracture surface of (a-1) PTB0.5PE0.1, (a-2) pr-A(PTB0.5PE0.1), (b-1) PTB0.5PE0.3, (b-2) pr-A(PTB0.5PE0.3), (c-1) PTB0.5PE0.5 and (c-2) $p r-\mathrm{A}(\mathrm{PTB} 0.5 \mathrm{PE} 0.5)$.

Fig. 7 Pore size distributions of (a) pr-A(PTB0.5P0.5), (b) pr-A(PTB0.5T0.5) and (c) pr-A(PTB0.5PE0.5).

Fig. 8 SEM photographs of fracture surface of (a) pr-A(PTB0.5P0.5) and (b) pr-A(PTB0.5T0.5).

Fig. 9 Variations in size of cell windows measured by using mercury porosimeter and open porosity $p r-\mathrm{A}(\mathrm{PTB} m \mathrm{Sn})$ with $n$ values.

Fig. 10 Variations in size of cell windows measured by using SEM photographs and mercury porosimeter and open porosity $\mathrm{pr}-\mathrm{A}(\mathrm{PTB} m \mathrm{Sn})$ with contact angle of Ncl-PMMA adhesive solutions. 
(a) РTBO

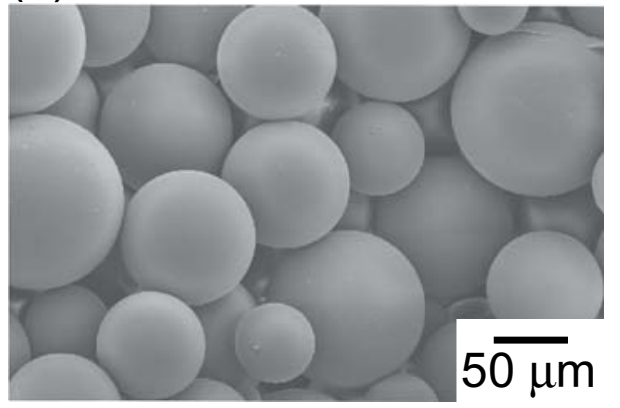

(d) PTB0.5

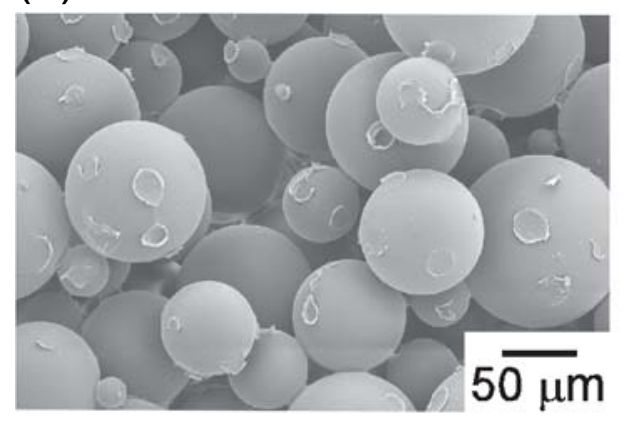

(b) PTB0.1

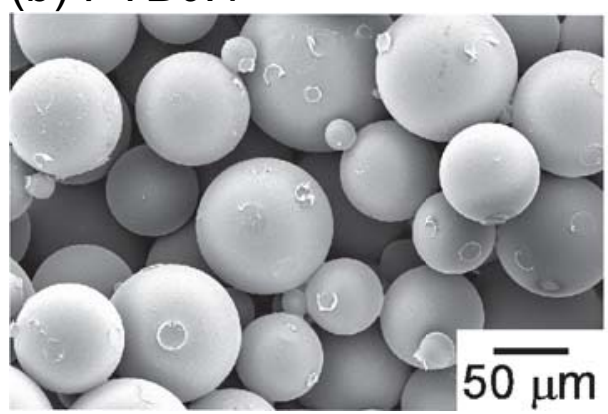

(e) PTB1.0

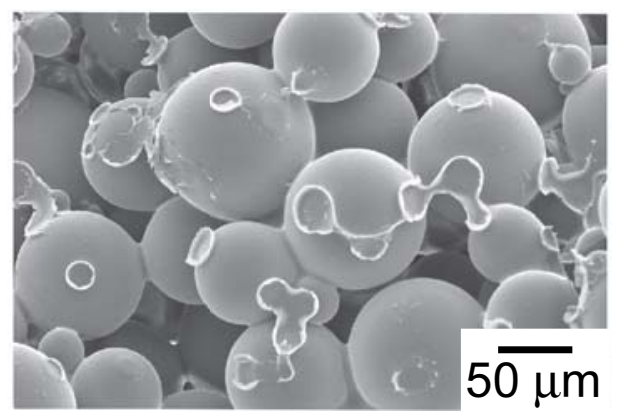

(c) PTB 0.3

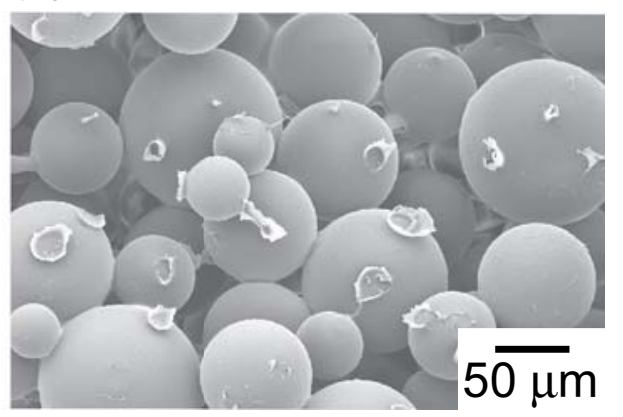

(f) PTB1.5

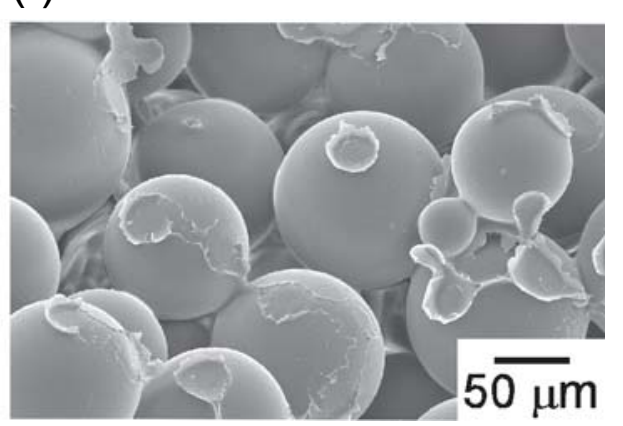

Fig. 1 Kamitani et al. 
(a) $p r-\mathrm{A}(\mathrm{PTB} 0)$

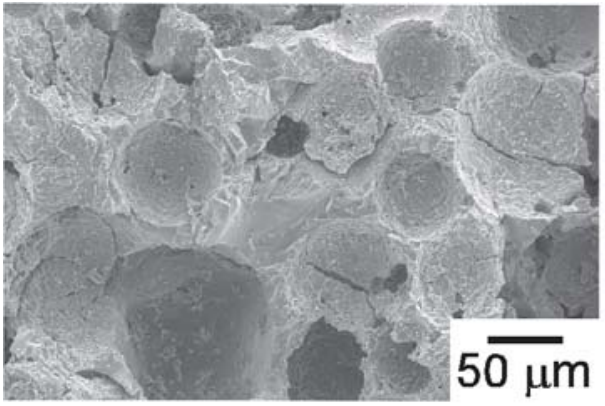

(d) $p r-A($ PTB0.5)

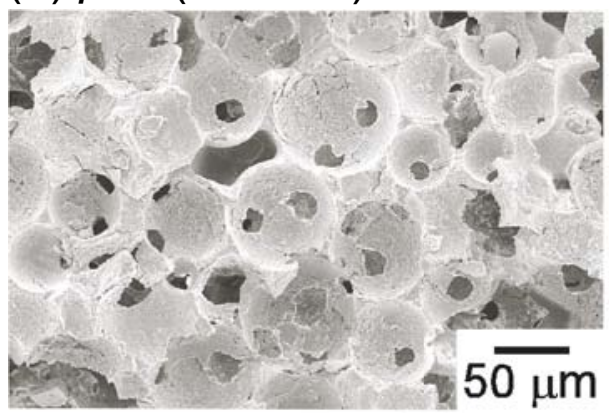

(b) $p r-\mathrm{A}(\mathrm{PTB} 0.1)$

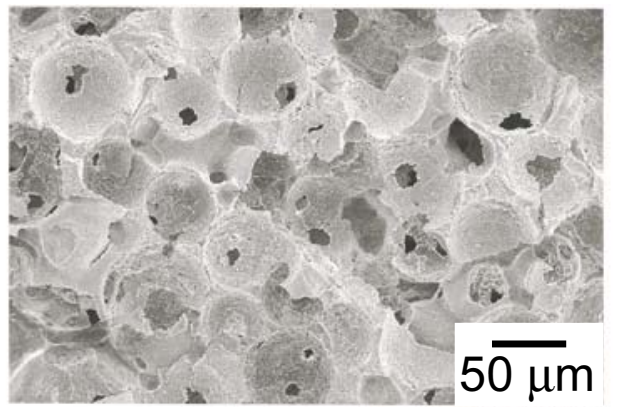

(e) $p r-A(P T B 1.0)$

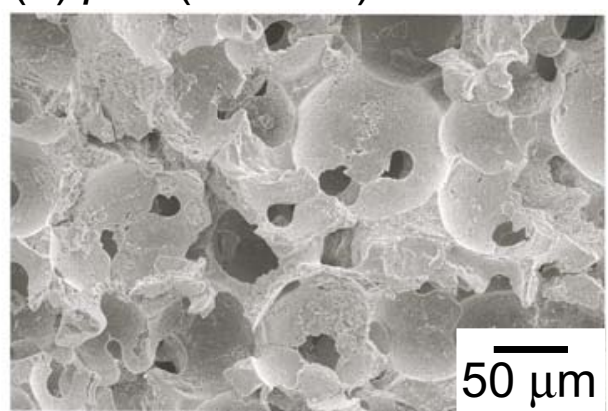

(c) $p r-\mathrm{A}(\mathrm{PTB} 0.3)$

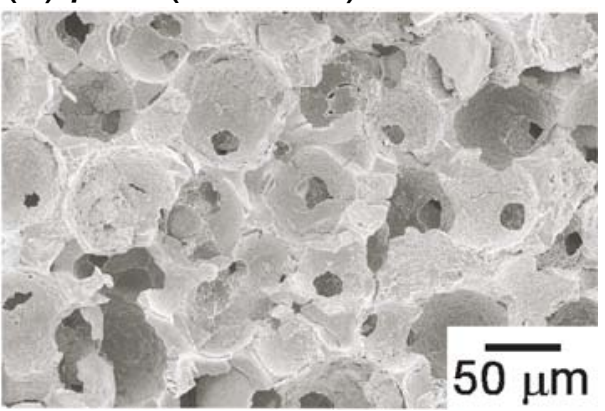

(f) $p r-\mathrm{A}(\mathrm{PTB} 1.5)$

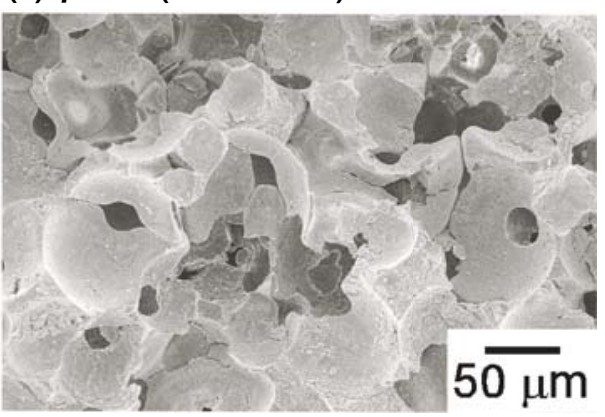

Fig. 2 Kamitani et al. 


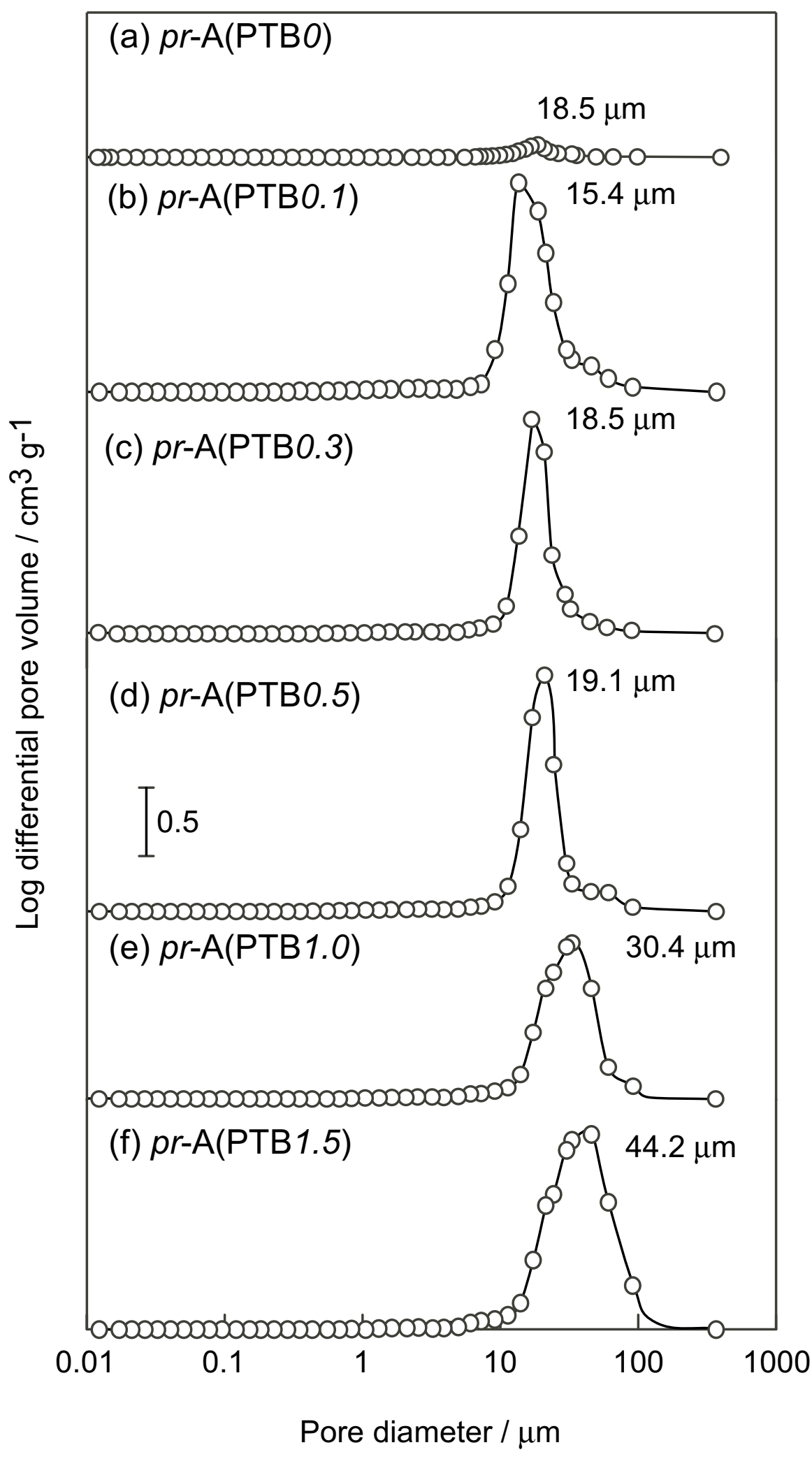

Fig. 3 Kamitani et al. 


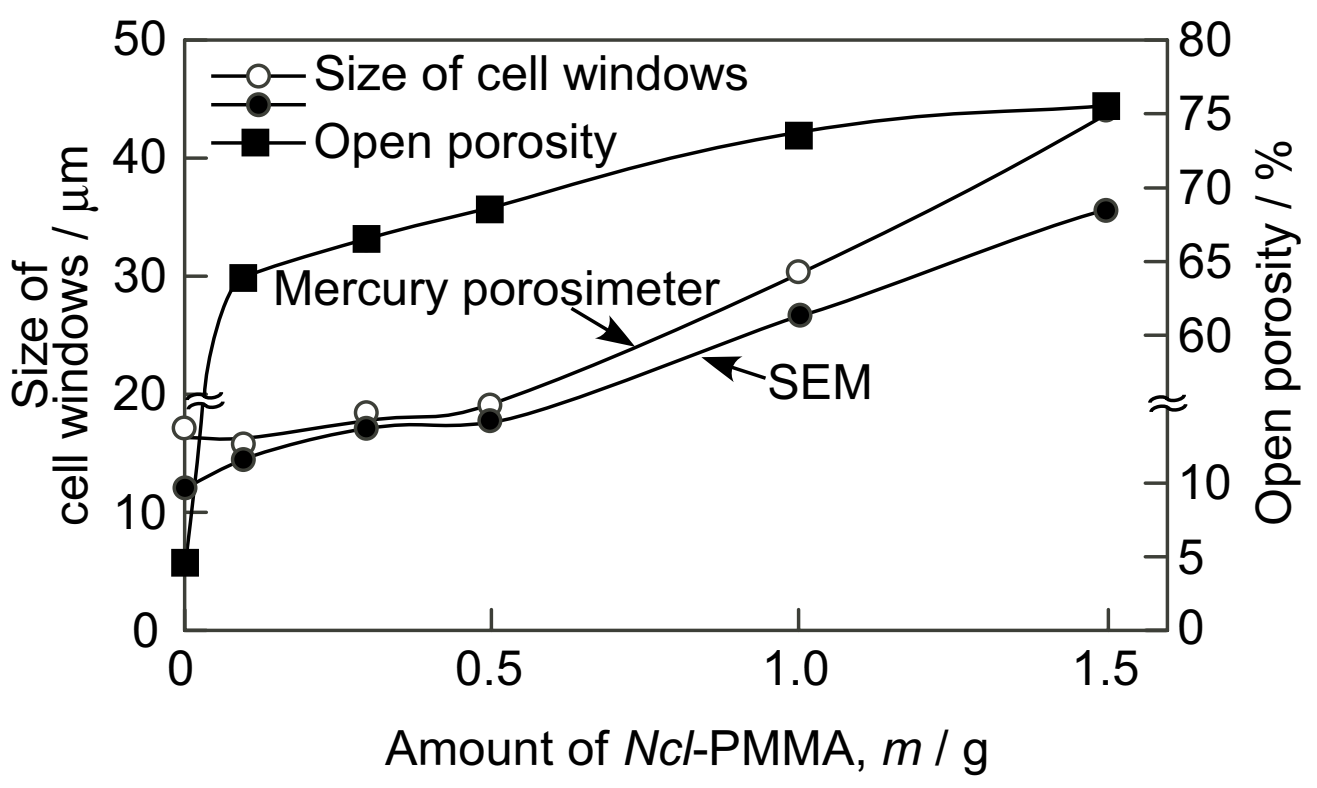

Fig. 4 Kamitani et al. 


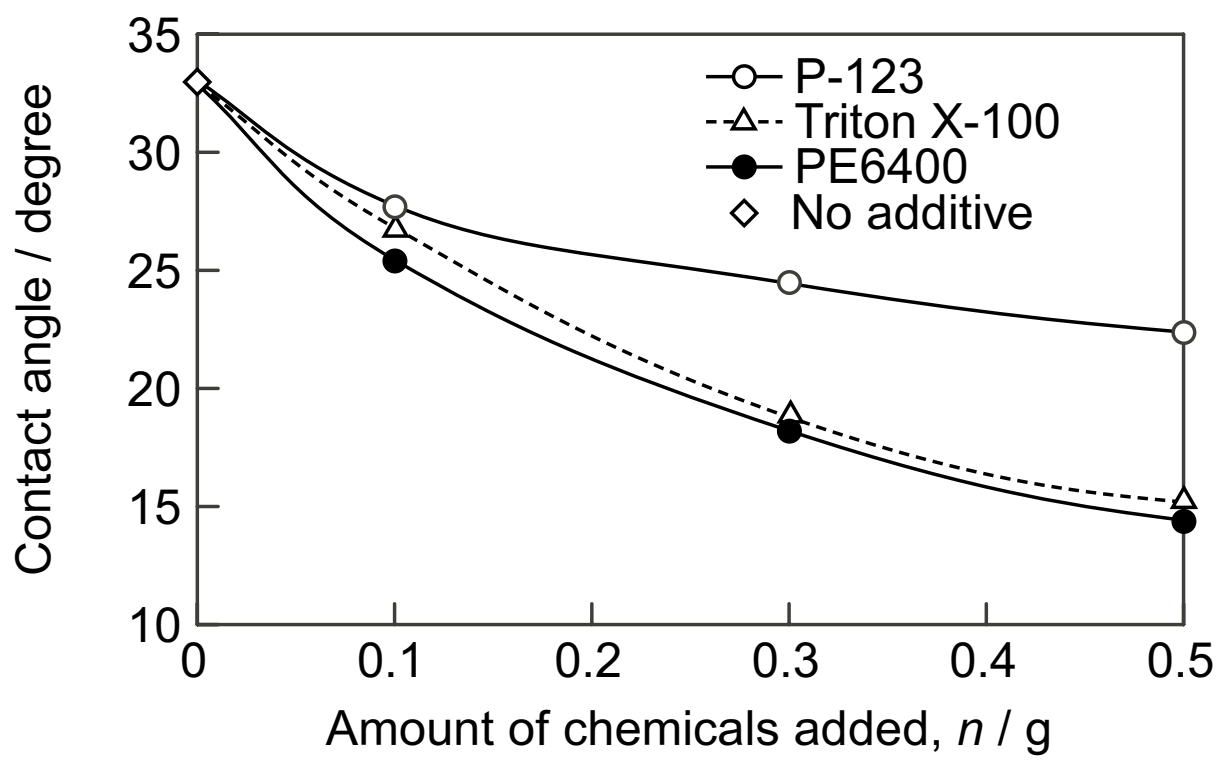

Fig. 5 Kamitani et al. 
(a-1) PTB0.5PE0.1

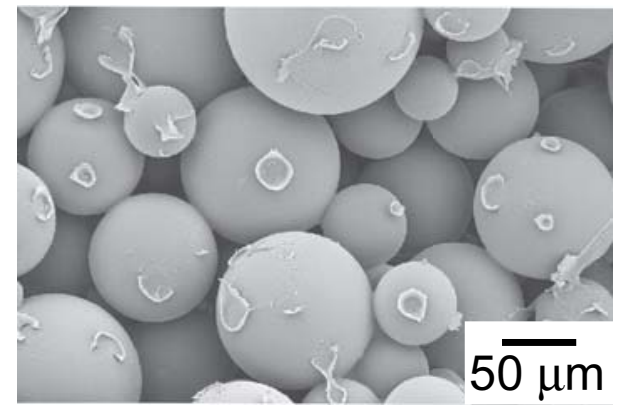

(b-1) PTB0.5PE0.3

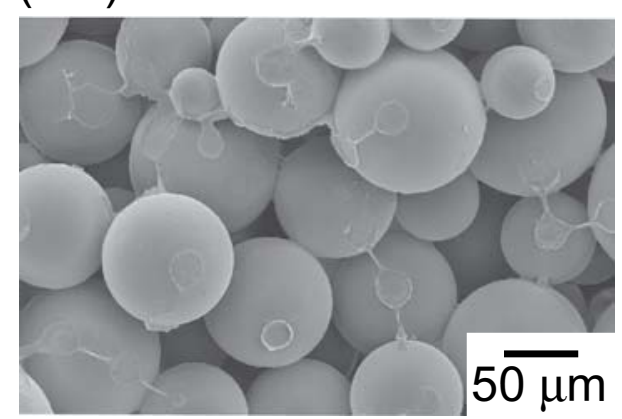

(c-1) PTB0.5PE0.5

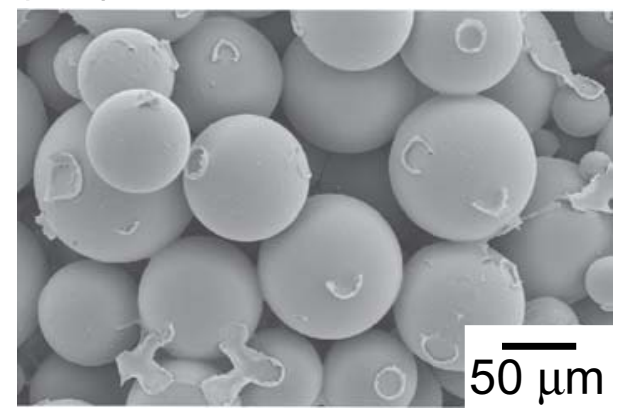

(a-2) pr-A(PTB0.5PE0.1)

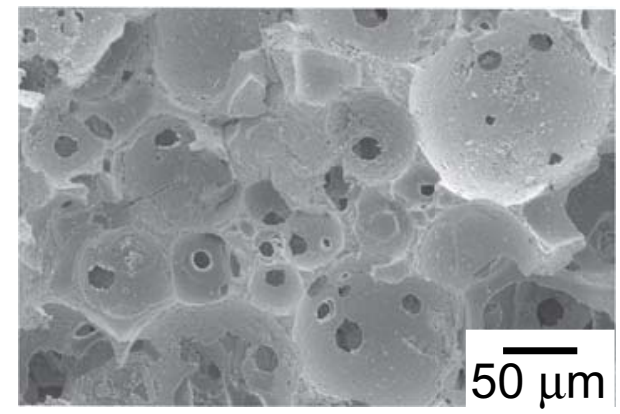

(b-2) pr-A(PTB0.5PE0.3)

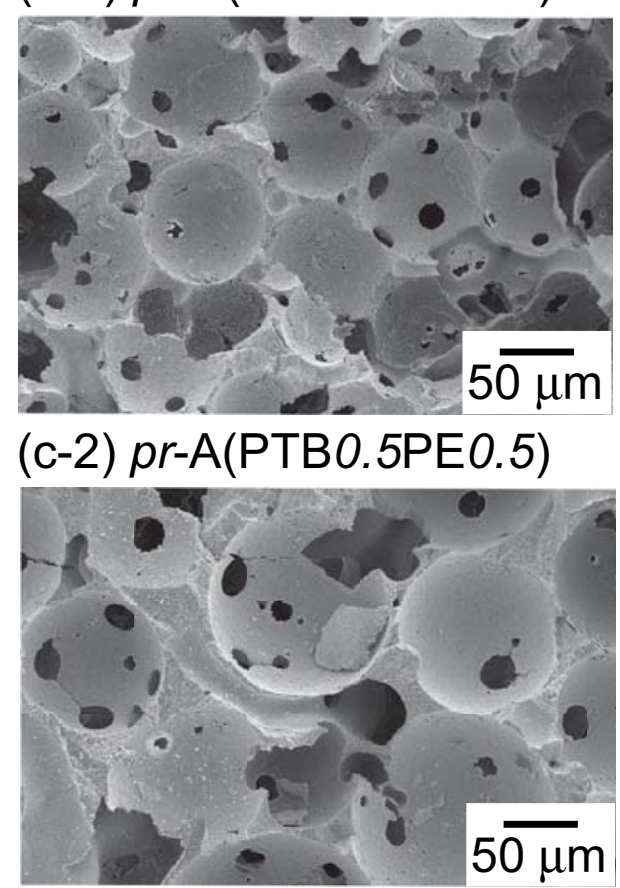

Fig. 6 Kamitani et al. 


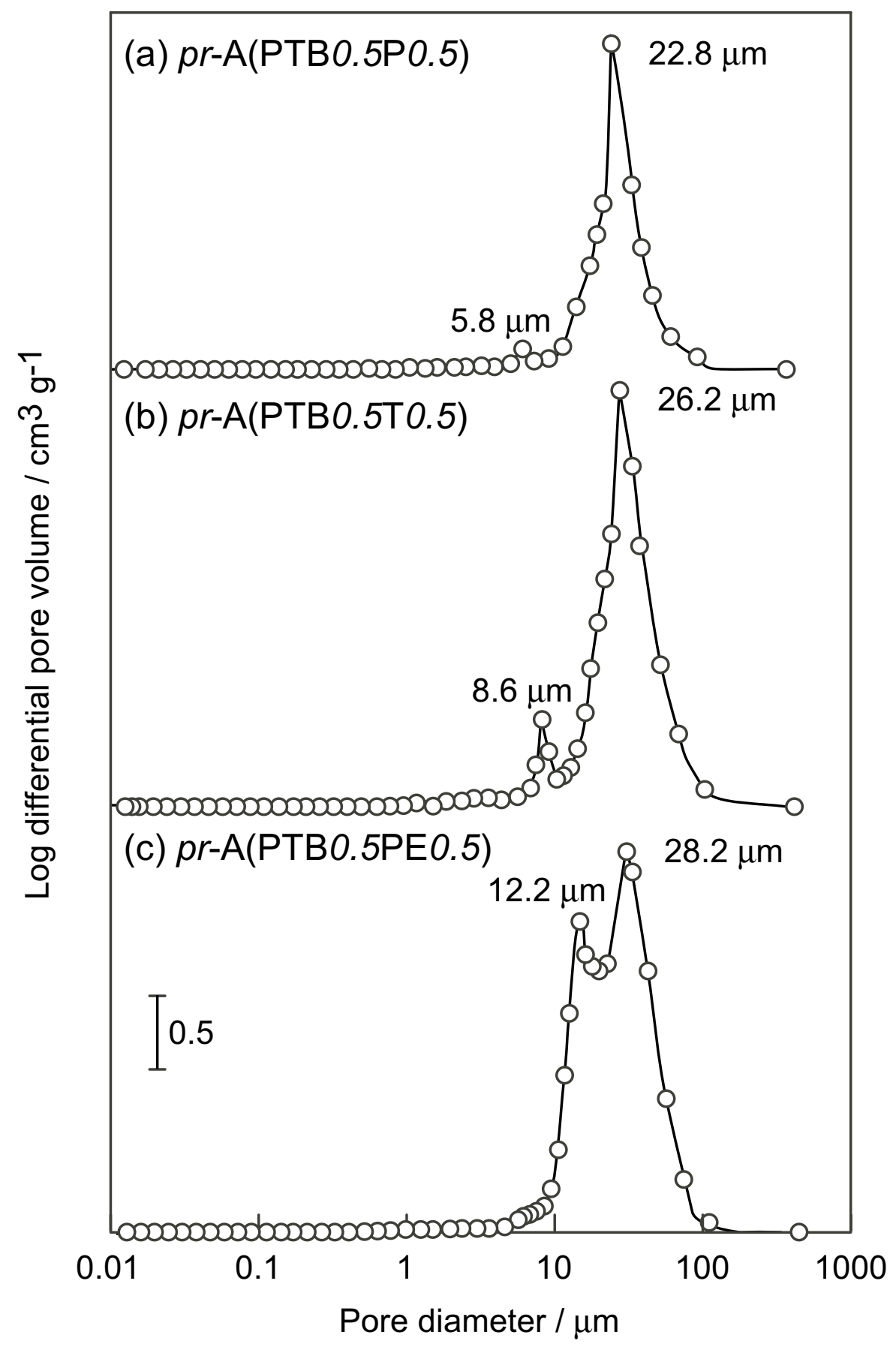

Fig. 7 Kamitani et al. 
(a) $p r-\mathrm{A}(\mathrm{PTB} 0.5 \mathrm{P} 0.5)$

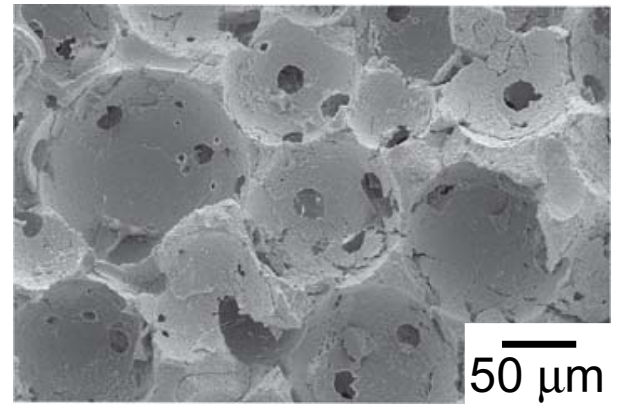

(b) $p r-\mathrm{A}(\mathrm{PTB} 0.5 \mathrm{~T} 0.5)$

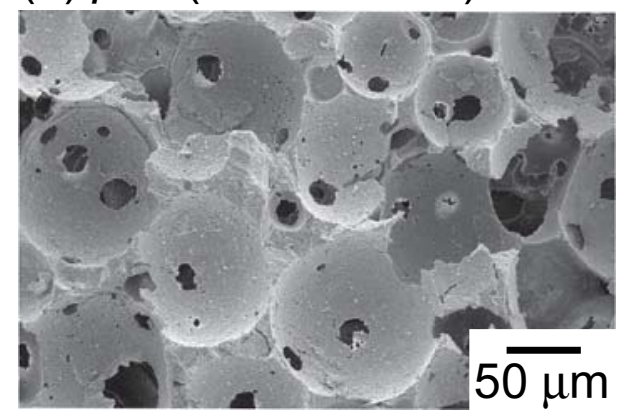

Fig. 8 Kamitani et al. 


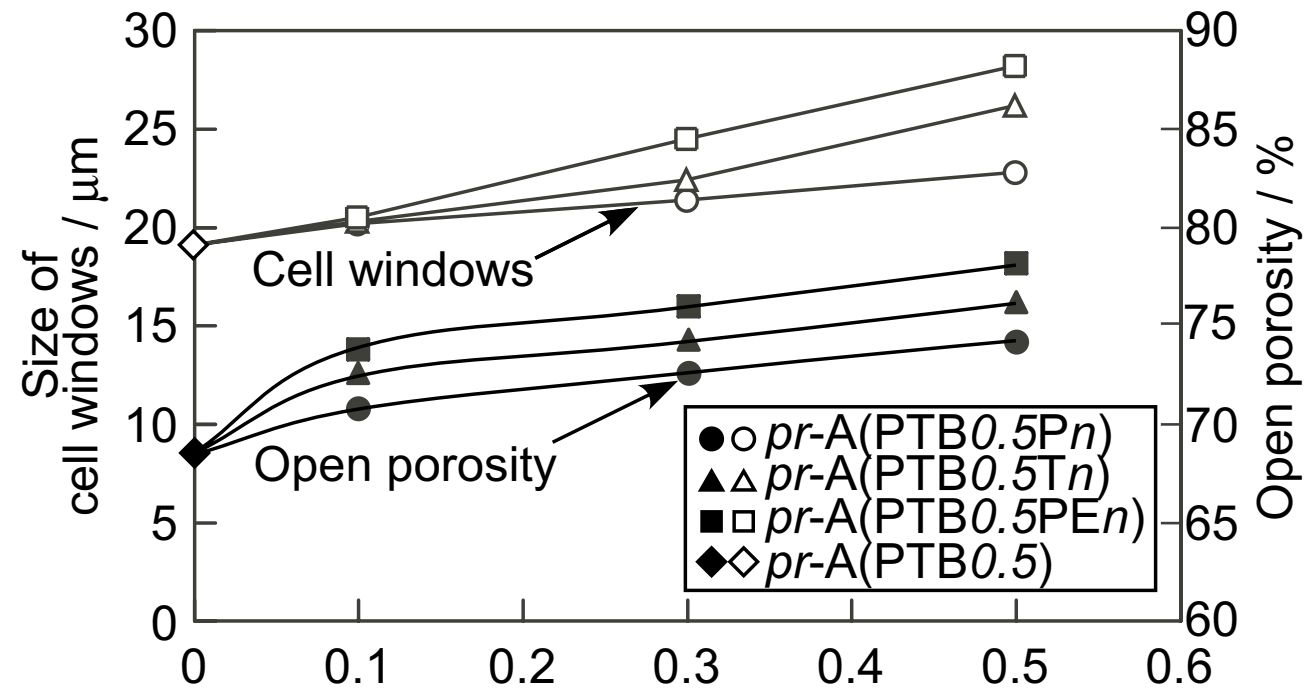

Amount of chemicals added, $n / \mathrm{g}$

Fig. 9 Kamitani et al. 


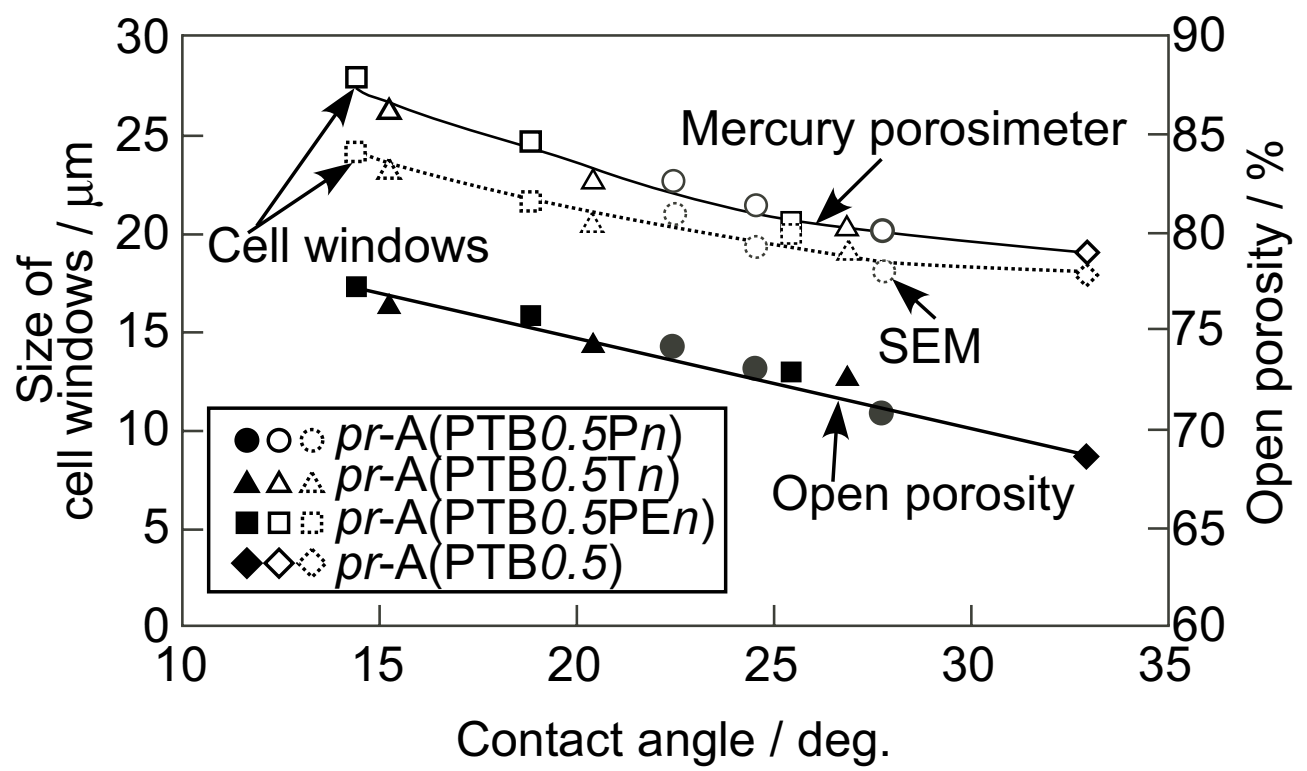

Fig. 10 Kamitani et al. 